\title{
Design and Construction of a Flight Data Acquisition System Adapted to an Experimental Rocket Model
}

\author{
Diseño y Construcción de un Sistema de Adquisición de Datos de Vuelo Adaptado a un \\ Modelo de Cohetería Experimental
}

\section{J. Alejandro Urrego P. (1) 1* | Luisa Mónico (10 1* | Harold Acosta (1) $1^{1^{*}}$ | Germán Reyes ${ }^{1^{*}}$ | Luis Hernández ${ }^{1^{*}}$}

${ }^{1}$ Programa de Ingeniería Aeronáutica. Facultad de Ingeniería. Universidad de San Buenaventura, sede Bogotá-Colombia.

\section{Correspondence}

Alejandro Urrego, Profesor asociado Programa de Ingeniería Aeronáutica. Facultad de Ingeniería. Universidad de San Buenaventura, sede Bogotá-Colombia. Email: jurrego@usbbog.edu.co

Copyright : Licencia de Creative Commons Reconocimiento-NoComercial 4.0 Interna.

\section{@creative}

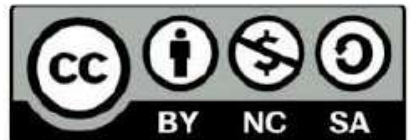

Editors: Robert Paul Salazar

Editorial assistant : Luz Adriana Suáres Suáres.

How to cite

Urrego A. et al., Diseño y Construcción de un Sistema de Adquisición de Datos de Vuelo Adaptado a un Modelo de Cohetería Experimental, TECCIENCIA, Vol. 14, No. 27, 73-83, 2019

DOI:http://dx.doi.org/10.18180/tecciencia.2019.27.8

\section{1 | INTRODUCCIÓN}

En este documento se presenta el diseño, construcción y caracterización de un dispositivo de adquisición de datos de vuelo para el cohete USB - Rocket I, el cual fue construido y ensamblado en la Universidad de San Buenaventura, sede Bogotá. El objetivo principal de este proyecto radica en la ausencia de un dispositivo de

*Equally contributing authors.

ABSTRACT. This document describes the different stages of development need to design, build and characterize a flight data acquisition system for the experimental rocket USB - Rocket I. To do so, some simulated flight conditions collected from a computer program, and the manufacturing and assembly process of an integrated electronic circuit are presented. As a result, the flight data collected in a horizontal ground displacement and commercial flight test are presented with this device.

keywords: Data acquisition, Experimental rocketry, Solid fuel, Raspberry Pi, Micro-electro-mechanical systems.

RESUMEN. En este documento se presentan las etapas de desarrollo realizadas para el diseño, construcción y caracterización de un sistema que permita la adquisición de datos de vuelo del cohete experimental USB - Rocket I. Para ello, se exponen las condiciones obtenidas de la simulación de vuelo, empleando un programa especializado de cohetería, como también el proceso de construcción e integración de sensores en un circuito electrónico, el cual permite la adquisición de datos. Como resultado, se presentan las mediciones obtenidas con el sistema durante una prueba en condición de desplazamiento horizontal y al equipar una aeronave comercial con este dispositivo.

Palabras clave: Adquisición de datos, Cohetes experimentales, Combustible sólido, Raspberry Pi, Sistemas micro-electromecánicos. 
esta clase, que sea manufacturado y validado en el entorno académico, puesto que en el campo de la cohetería experimental, los dispositivos de adquisición de datos son mayoritariamente de adquisición comercial.

Durante la revisión del estado del arte, donde se describe y analiza el lanzamiento de cohetes experimentales construidos en Colombia, se evidenció el empleo de sistemas de adquisición de datos y de telemetría comerciales [1][2]. Asimismo, se estableció que en diferentes trabajos enfocados al desarrollo de equipos de telemetría y de centros de control para cohetes experimentales, los cuales requerían la integración de componentes electrónicos [3] no se logró validar con éxito las condiciones de vuelo reales y simuladas en programas especializados de cohetería [4].

Por su lado, otros trabajos proporcionaron detalles acerca de los elementos que se pueden emplear en un sistema de adquisición de datos, incorporados a cohetes con gran altura de apogeo [5, 6], para monitorear variables de vuelo tales como presión, calor, temperatura o deflexión de elementos mecánicos [7]. Del mismo modo, se evidenció el uso de sistemas GPS [8, 9], elementos tipo altímetro, sensores para la medición de temperatura, presión atmosférica y aceleración [10].

Es así como en este trabajo se planteó el desarrollo de un dispositivo que permita realizar la toma de datos de variables atmosféricas, como presión y temperatura, como también parámetros de vuelo tales como altura, aceleración y/o posición geográfica. Para ello, se propone el diseño con base en las condiciones geométricas y estructurales del cohete experimental USB - Rocket I, considerando la propulsión con un motor de combustible sólido tipo $\mathrm{J}^{1}$.

\section{I CARACTERIZACIÓN DEL COHETE USB - ROCKET I Y SIMULACIÓN DE UN VUELO NORMAL}

El cohete USB - Rocket I, mostrado en la Fig. 1, es un cohete experimental desarrollado en la Universidad de San Buenaventura, sede Bogotá, e I cual cuenta con una longitud de 1.14 metros, un diámetro de fuselaje de 7.62 centímetros y un peso al despegue de $2.5 \mathrm{~kg}$, aproximadamente [12]. Adicionalmente, este vehículo no tripulado incorpora tres aletas fijas, de 9 centímetros de envergadura cada una, para la estabilización en vuelo. El cohete fue construido en un fuselaje de adquisición comercial, reforzado con material compuesto, y equipado con mamparos estructurales de la bahía de carga manufacturados en ABS mediante el método por deposición fundida.

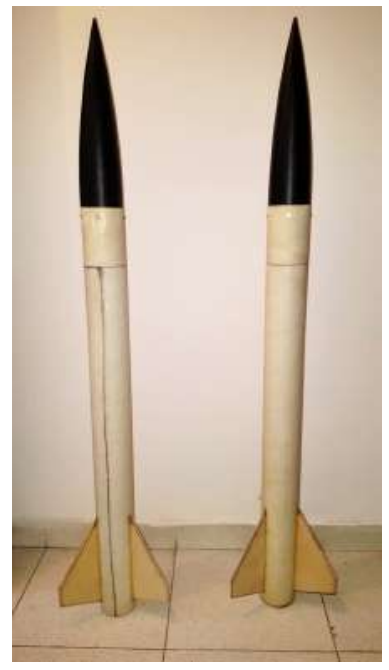

(a)

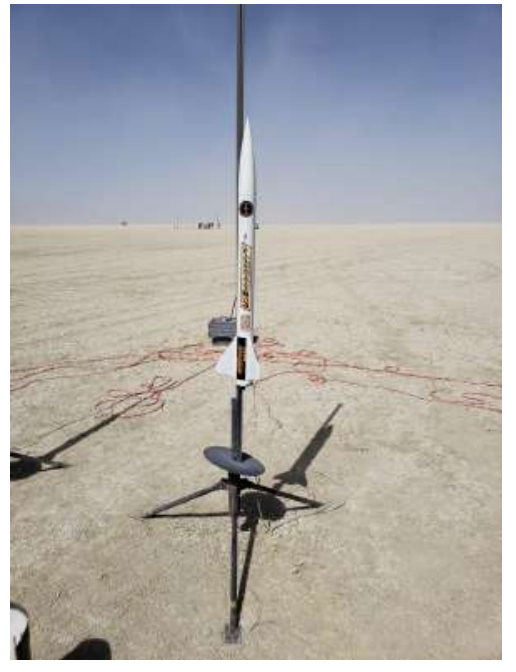

(b)

FIG. 1 (a) Cohete USB - Rocket I, (b) sobre la plataforma de lanzamiento.

\footnotetext{
${ }^{1}$ Actualmente en Colombia se llevan estudios experimentales para fomentar el uso de biocombustibles para aeronaves [11].
} 
Las etapas de vuelo de un cohete experimental son: despegue, ascenso propulsado por motor, ascenso en fase de vuelo inercial o no propulsado, apogeo en la altura máxima, descenso con paracaídas y aterrizaje. Para determinar las variables más importantes resultantes de vuelo del cohete, equipado con un motor sólido tipo J de referencia AeroTech J270W, se realizó la simulación de vuelo en el programa RockSim [13], permitiendo obtener las principales curvas características de vuelo: número de Mach, altura, aceleración, y otras [14]. Para ello, se consideraron las condiciones iniciales para la simulación que se muestran en la Tabla 1.

TABLA 2 Resultados principales obtenidos de la

TABLA 1 Condiciones iniciales propuestas para la simulación del cohete en RockSim. simulación del cohete en RockSim.

\begin{tabular}{|l|c|}
\hline PARÁMETRO & VALOR \\
\hline Altitud de lanzamiento & Nivel del mar \\
\hline Humedad relativa $(\%)$ & 50 \\
\hline Temperatura $\left({ }^{\circ} \mathrm{C}\right)$ & 25 \\
\hline Presión barométrica (bar) & 1.013 \\
\hline Velocidad del viento (km/h) & $0-4.667$ \\
\hline Ángulo de lanzamiento (grados) & 89 \\
\hline
\end{tabular}

\begin{tabular}{|l|c|}
\hline PARÁMETRO & VALOR \\
\hline Máximo número de Mach & 0.9 \\
\hline Máximo empuje del motor $(\mathrm{N})$ & 350.8 \\
\hline Tiempo al apagado del motor $(\mathrm{s})$ & 2.6 \\
\hline Aceleración máxima $\left(\mathrm{m} / \mathrm{s}^{2}\right)$ & 178.43 \\
\hline Velocidad máxima $(\mathrm{m} / \mathrm{s})$ & 303 \\
\hline Altura de apogeo $(\mathrm{m})$ & 2100 \\
\hline Tiempo de apogeo $(\mathrm{s})$ & 2079.52 \\
\hline Tiempo de vuelo $(\mathrm{s})$ & 407 \\
\hline Rango (m) & 250 \\
\hline Margen estático & $2.52-3.68$ \\
\hline
\end{tabular}

Los principales resultados de la simulación en el programa RockSim se muestran en la Tabla 2. En la Fig. 2, se presenta la variación del número de Mach durante el tiempo de vuelo, desde la fase de ascenso del cohete hasta la apertura del paracaídas en la fase apogeo. Se observa un aumento gradual del número de Mach durante $2.55 \mathrm{~s}$, tiempo en el cual el motor finaliza la fase de tiempo de quemado de combustible; Es así como el máximo valor alcanzado durante esta fase de vuelo propulsado se registra en $2.31 \mathrm{~s}$ con Mach 0.90. Asimismo, se evidencia una altura máxima alcanzada de $2079 \mathrm{~m}, 1330 \mathrm{~m}$ por encima de la altura máxima lograda al equipar el cohete con motores con un impulso total $230 N-s$.

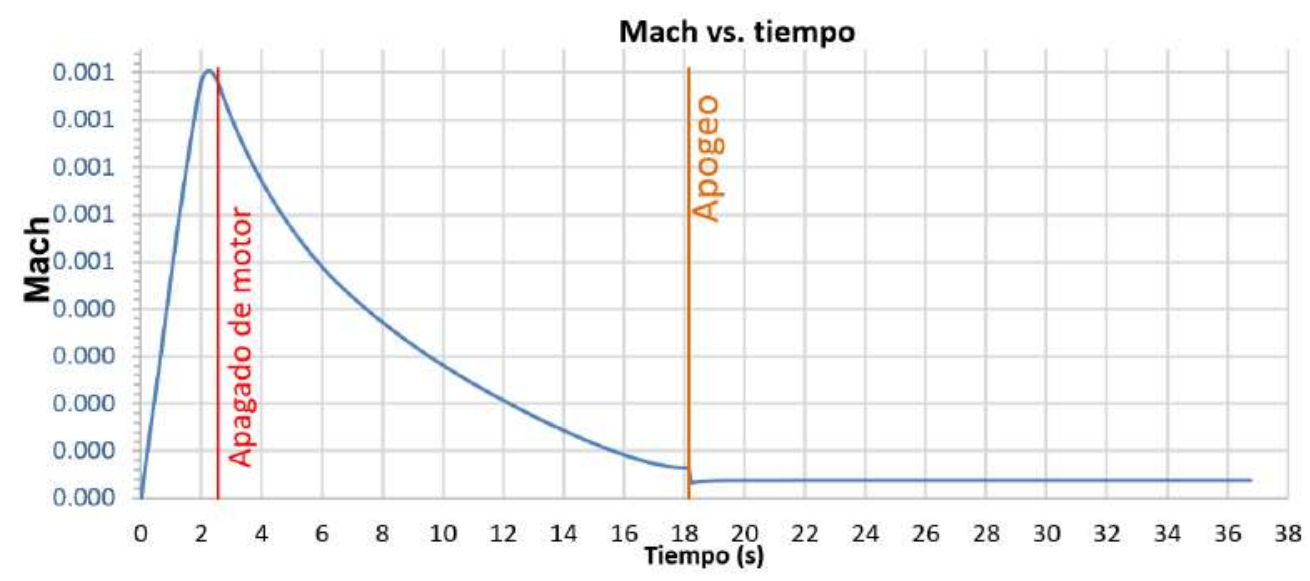

FIG. 2 Número de Mach del cohete obtenido en la simulación.

En la Fig. 3 se presenta la gráfica de la altura, velocidad y aceleración de vuelo del cohete experimental. Se observa que la fase de apogeo se alcanza aproximadamente a los 17 segundos de vuelo, con una altura máxima de $2079 \mathrm{~m}$ y una aceleración no mayor a $178.43 \mathrm{~m} / \mathrm{s}^{2}$. En el tiempo comprendido entre la fase de encendido y el máximo empuje entregado por el motor se registra una fluctuación de la aceleración entre $178.4 \mathrm{~m} / \mathrm{s}^{2}$ y $185.9 \mathrm{~m} / \mathrm{s}^{2}$, y un incremento lineal de la velocidad del vehículo hasta un valor de $118.70 \mathrm{~m} / \mathrm{s}$. Una vez el motor presenta su empuje máximo, se evidencia una reducción exponencial de la aceleración de $185.90 \mathrm{~m} / \mathrm{s}^{2}$ 
a $39.62 \mathrm{~m} / \mathrm{s}^{2}$ en $2.55 \mathrm{~s}$, tiempo en el cual el motor quema por completo su combustible y el cohete entra en la fase de vuelo inercial [12].

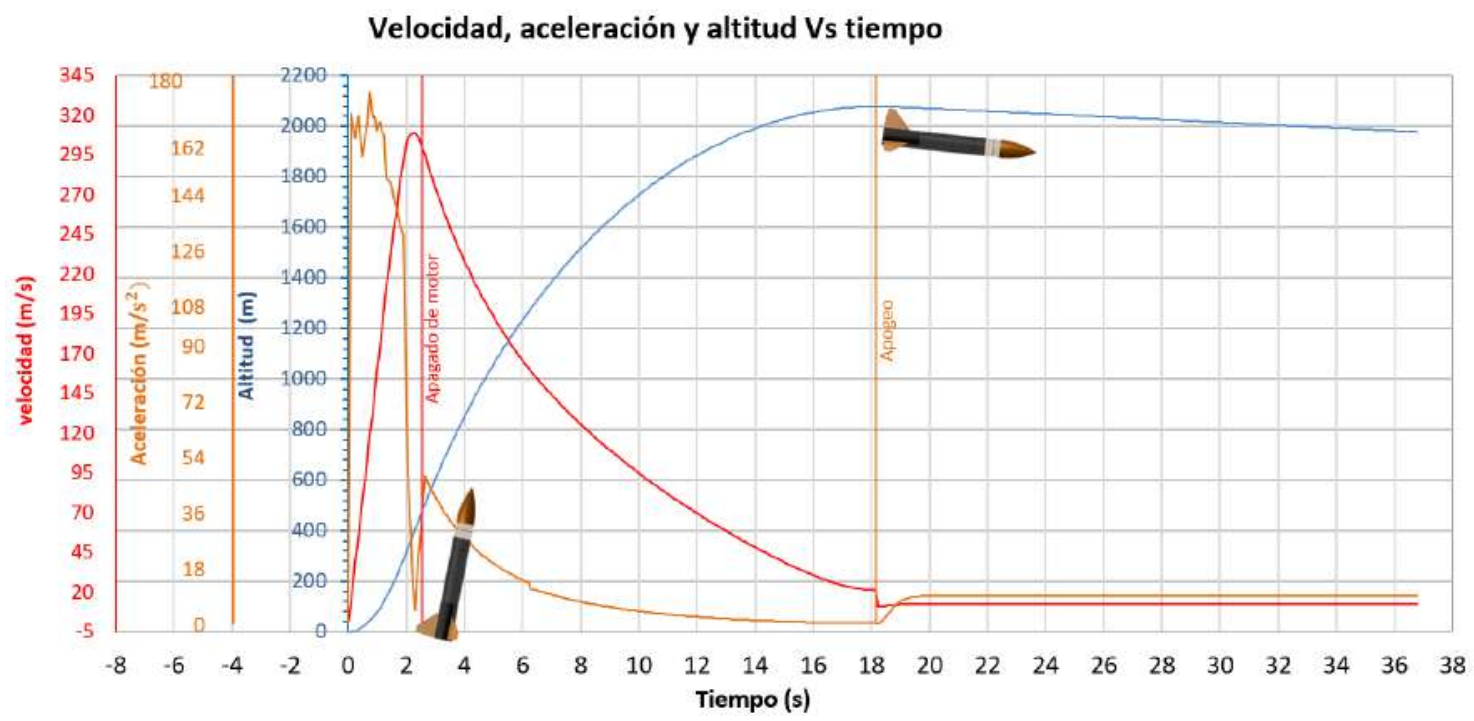

FIG. 3 Comportamiento de la altura de vuelo obtenido en la simulación.

Del mismo modo, durante esta fase se registró un incremento continuo de la velocidad hasta encontrar un valor máximo de $303 \mathrm{~m} / \mathrm{s}$ y una altura de $481.70 \mathrm{~m}$. Una vez el cohete entra en la fase de vuelo inercial a los $2.55 \mathrm{~s}$, se evidenció una disminución gradual de la aceleración hasta la fase de apogeo, con un valor de $0.26 \mathrm{~m} / \mathrm{s}^{2}$ en $18.15 \mathrm{~s}$, tiempo en el que el cohete alcanza su altura máxima de $2079.52 \mathrm{~m}$ e inicia la etapa de caída libre. Del mismo modo, en esta fase se registra una velocidad de $21.15 \mathrm{~m} / \mathrm{s}$ y un mach mínimo de 0.033 . Es importante resaltar que la fase de apogeo, etapa en la cual el cohete presenta regímenes de estabilidad constantes, se evidencia nuevamente un incremento gradual de la aceleración de $0.26 \mathrm{~m} / \mathrm{s}^{2}$ en $18.1 \mathrm{~s}$, a $9.75 \mathrm{~m} / \mathrm{s}^{2}$ en $21.2 \mathrm{~s}$, tiempo aproximado en el cual el paracaídas se despliega y comienza la fase de recuperación del vehículo. Del mismo modo, se evidencia una velocidad de $12.3 \mathrm{~m} / \mathrm{s}$ en $21.1 \mathrm{~s}$ y una disminución paulatina hasta $8.5 \mathrm{~m} / \mathrm{s}$ en $407 s$, tiempo de aterrizaje del cohete.

\section{1 | Diseño del circuito de adquisición de datos}

El proceso de diseño para el circuito electrónico de adquisición de datos se realizó en tres partes: en primer lugar, se definieron las variables para la medición y los requerimientos del equipo. Con base en esto, se seleccionaron los diferentes componentes del sistema. Finalmente, se realizó el trabajo de integración, el diseño del circuito, la manufactura de una placa impresa y la verificación del funcionamiento de los elementos. Los requerimientos propuestos para el sistema se listan a continuación:

- Integración correcta con las características geométricas de la bahía de carga y el fuselaje del cohete USB - Rocket I.

- Resistencia estructural suficiente ante las condiciones de carga generadas por la aceleración generada durante el despegue (ver Fig. 3).

- $\quad$ Almacenamiento de los datos medidos, para el posterior procesamiento.

Además, se establecieron las siguientes variables a adquirir durante un vuelo del cohete experimental:

- $\quad$ Temperatura de la bahía de carga que aloja el sistema. 
- $\quad$ Presión atmosférica a la altura de vuelo.

- $\quad$ Aceleración, velocidad y altura del cohete durante el vuelo.

- Posición del vehículo tipo cohete en coordenadas geográficas.

Una vez propuestas las condiciones de medición, se determinaron los siguientes elementos para la adquisición de parámetros:

- $\quad$ Sensor de temperatura y presión atmosférica BMP180, el cual garantiza un rango de medición de 300 hasta $1100 \mathrm{hPa}$, dentro de un rango de temperatura de $-40 \mathrm{a}$ $85^{\circ} \mathrm{C}$, con una resolución de $\pm 1 \mathrm{~m}$ e n el cálculo de la altura [15]

- Unidad de medición inercial (IMU) MPU6050, con un rango de medición de \pm 16 $\mathrm{g}$ para la aceleración, y un rango de medición para la velocidad angular de hasta \pm $2000 \%$ s.

- Acelerómetro LIS331, que garantiza un rango de operación de $\pm 24 g$, permitiendo la operación de este sensor dentro de las características operativas del cohete.

- $\quad$ GPS L86 [16], el cual asegura una precisión menor a 2.5m en la determinación de la posición geográfica.

- $\quad$ La integración de todos los sensores se realizó sobre una Raspberry Pi 3 B+ [17], empleando el sistema operativo Raspbian.

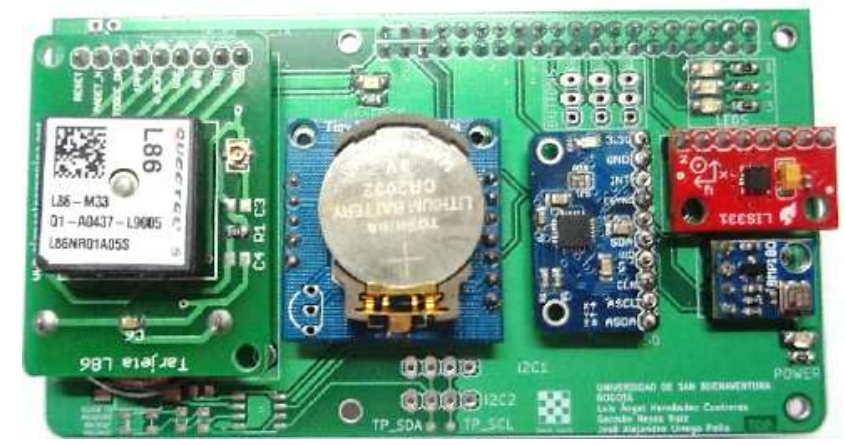

FIG. 4 Placa para integración de elementos de medición.

Asimismo, se realizó en KiCad [18] el diseño de una placa para circuito impreso que facilitara la integración de los componentes con la Raspberry Pi [12] y la adaptación a la estructura del cohete. En la placa se consideraron todos los elementos descritos con anterioridad, además de diferentes espacios para botones, dispositivos $I^{2} C$ adicionales e indicadores LED. Cabe resaltar que la alimentación del sistema se realizó empleando dos baterías de ion litio de 3.7 voltios y $3000 \mathrm{mAh}$ cada una. En la Fig. 4 se presenta la placa de sensores del trabajo con la integración completada.

\section{2 | Desarrollo de servidor para verificación de parámetros en fase pre-vuelo}

Previo al vuelo de prueba del cohete experimental, es importante la verificación de todos los sensores del sistema de adquisición de datos, verificando que las mediciones se tomen de manera correcta. Para tener acceso a las mediciones realizadas por el sistema desarrollado en este trabajo, se desarrolló un servidor web y una interfaz gráfica, que emplean la red de conexión WiFi creada por la Raspberry Pi 3 B+.

El protocolo de comunicación empleado para la transmisión de datos es de tipo cliente/servidor por medio 
de una conexión tipo websocket [19]. Se tiene un servidor, en este caso la Raspberry Pi; y un cliente, en este caso, un cliente con interfaz HTML ejecutado desde un dispositivo que deberá estar conectado a la red inalámbrica de la Raspberry Pi. Los datos enviados por el servidor, a través de la red inalámbrica, hasta el cliente son:

- Datos de altura por presión atmosférica y temperatura de la bahía de carga, obtenidos por el sensor BMP180.

- $\quad$ Datos de aceleración y altura por aceleración, obtenidos de la IMU MPU6050 y del acelerómetro LIS331.

- $\quad$ Altura, latitud, longitud y número de satélites conectados al sistema de GPS del equipo de adquisición de datos.

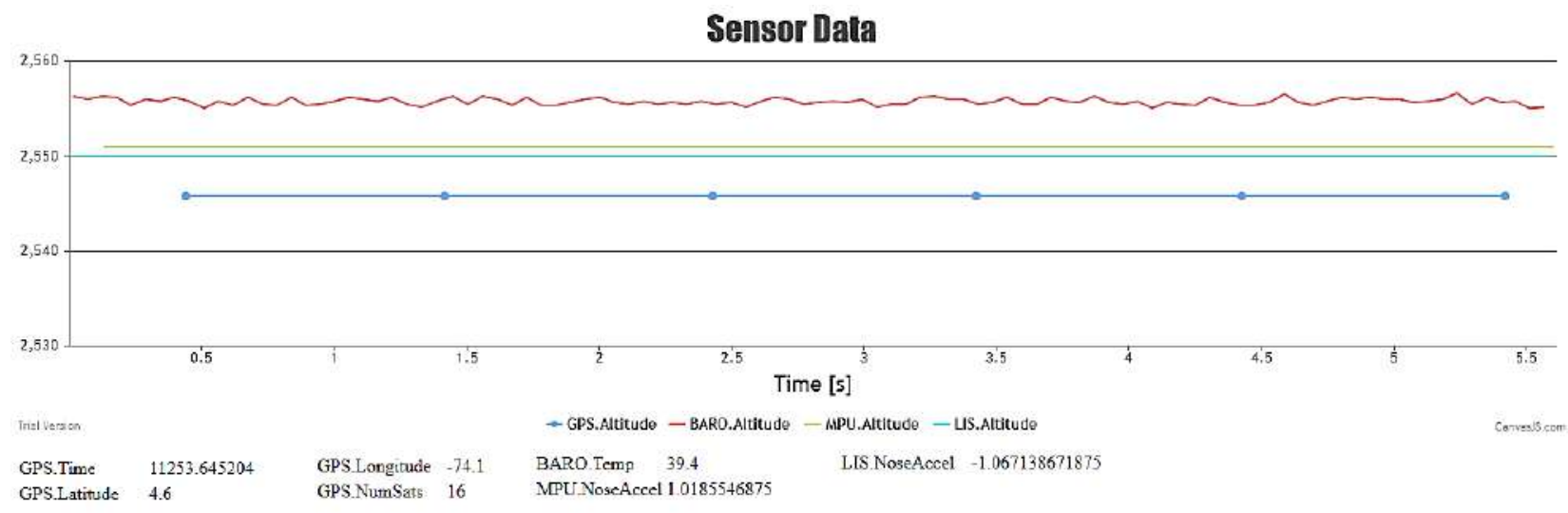

FIG. 5 Interfaz gráfica del servidor HTML para el monitoreo de los principales parámetros de medición, previo al vuelo del cohete.

En la Fig. 5, se muestra la interfaz gráfica del cliente web desarrollado, y se presenta la capacidad del mismo para mostrar los parámetros de vuelo.

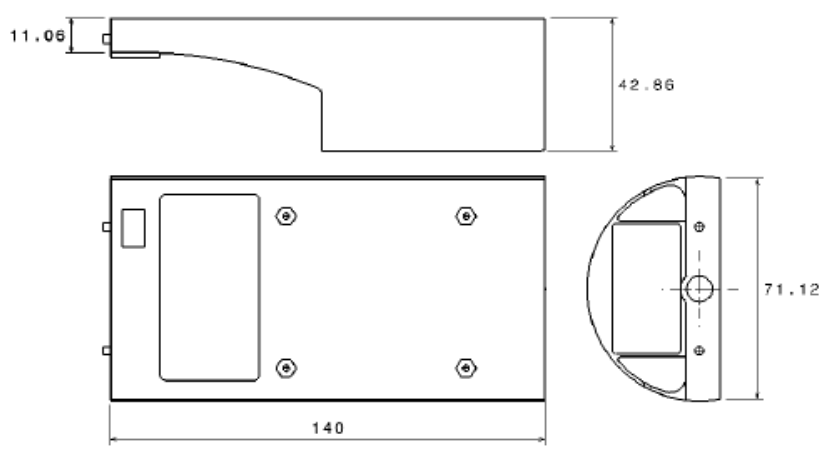

FIG. 6 Esquema del diseño de la base de soporte para el prototipo (unidades en $\mathrm{mm}$ ).

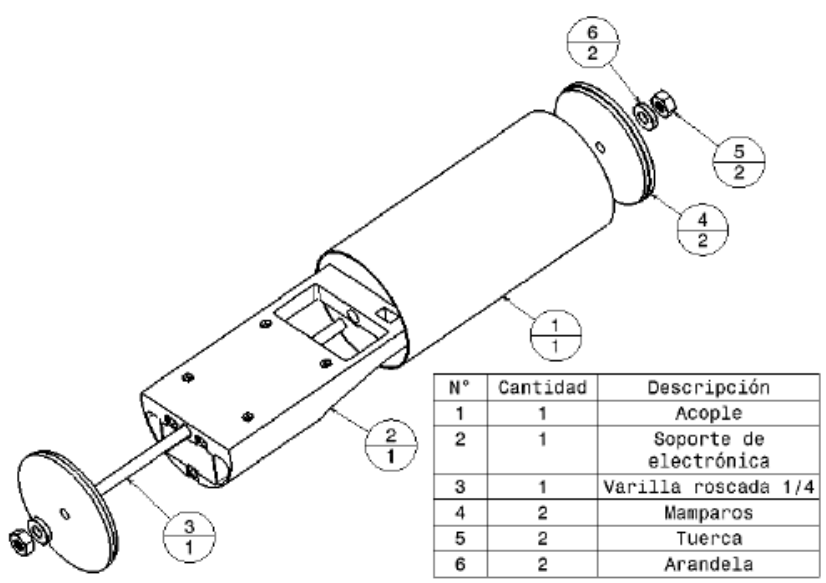

FIG. 7 Montaje explosionado de los principales elementos que conforman la bahía de carga del cohete USB - Rocket I. 


\section{3 | Construcción de bahía de carga para soporte del sistema de medición electrónica de datos}

Como soporte para incorporar los elementos de medición a la bahía de carga del cohete, se realizó el diseño y manufactura de una base y la adaptación de la bahía de carga. La base de soporte para la electrónica se diseñó en CATIA V5, y se modeló la geometría que se muestra en la Fig. 6. La manufactura de esta base se realizó mediante la construcción por manufactura aditiva FDM (Fused Deposition Modeling) empleando ABS (Acrilonitrilo Butadieno Estireno).

Por otra parte, la bahía de carga electrónica del cohete está conformada por los siguientes elementos: una sección de tubo de fuselaje, un tubo de acople, dos mamparos estructurales, una varilla roscada, elementos de sujeción (tuercas y arandelas). Así, se obtiene el sistema que se observa en la Fig. 7. El tubo de fuselaje del sistema se construyó a partir de un tubo comercial (LOC Coupler) que se reforzó con material compuesto en fibra de vidrio y resina epóxica, para obtener un espesor adecuado y garantizar la resistencia estructural suficiente. Además, al tubo de fuselaje se le realizó un proceso de acabado para obtener una superficie lisa que minimice la afectación en la aerodinámica durante el vuelo.

\section{4 | Pruebas de funcionamiento del prototipo}

Se realizaron dos pruebas para demostrar la capacidad de adquisición de datos del sistema desarrollado en este trabajo de investigación: la primera, se realizó en una condición de movimiento horizontal en automóvil; la segunda, en un vuelo en aeronave comercial en la ruta Medellín - Bogotá. En esta sección se presentarán y analizarán los resultados más importantes de estas dos pruebas.

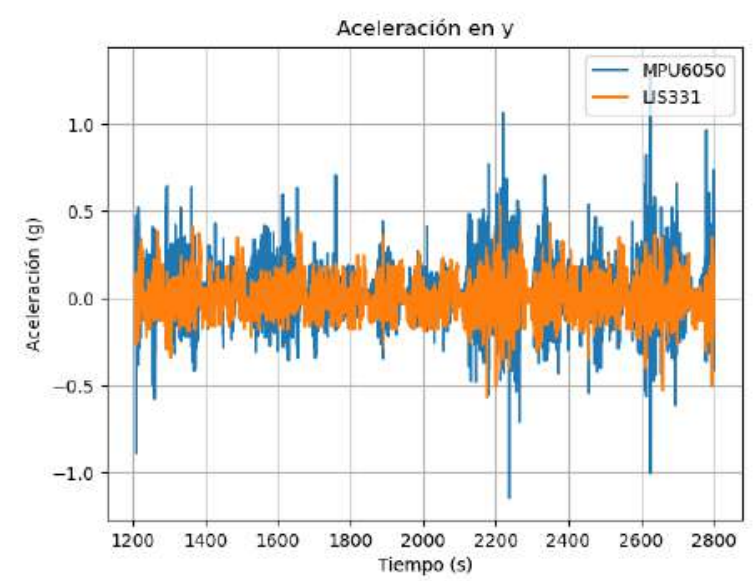

FIG. 8 Aceleración horizontal del vehículo en la dirección del movimiento - Prueba en tierra.

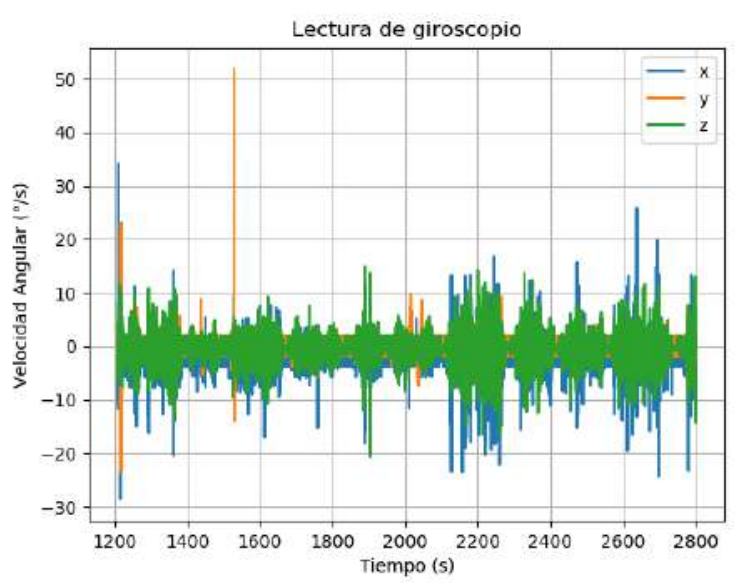

FIG. 9 Mediciones de velocidad angular - Prueba en tierra.

En primer lugar, como se observa en la Fig. 8, la aceleración registrada por los dos acelerómetros del sistema durante la prueba de desplazamiento horizontal en tierra. Se observa una variación entre los valores de $\pm 1 \mathrm{~g}$. Además, se puede determinar, a partir de la gráfica, que el sensor MPU6050 presenta una mayor variación en los datos, respecto a las mediciones registradas por el sensor LIS331, que presenta una variación menor. Se determina también que los valores registrados por el sistema son coherentes con el entorno de simulación en el que se desarrolló la prueba.

En la Fig. 9 se graficaron las mediciones de la velocidad angular registradas por el giroscopio de la IMU MPU6050 durante la prueba. La velocidad angular oscila mayoritariamente entre $\pm 10 \%$, ya que no se presentan cambios repetitivos en la posición angular del equipo de medición durante el experimento.

Las mediciones de la altura sobre el nivel del mar, registradas por el barómetro y por el GPS, se presentan en la Fig. 10. Se evidencia en la gráfica que las mediciones mantienen la misma tendencia y comportamiento; sin embargo, existe una diferencia de aproximadamente 20 metros entre ambas mediciones. Esto se explica 


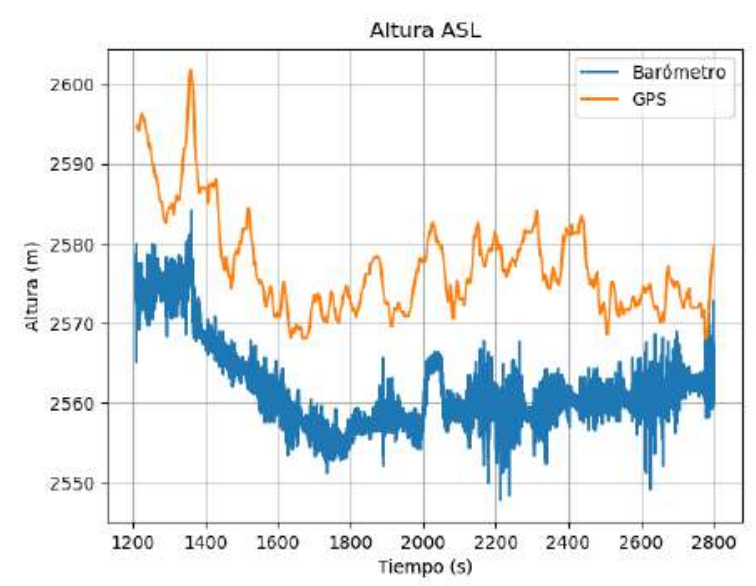

FIG. 10 Altura respecto al nivel del mar-Prueba en tierra.
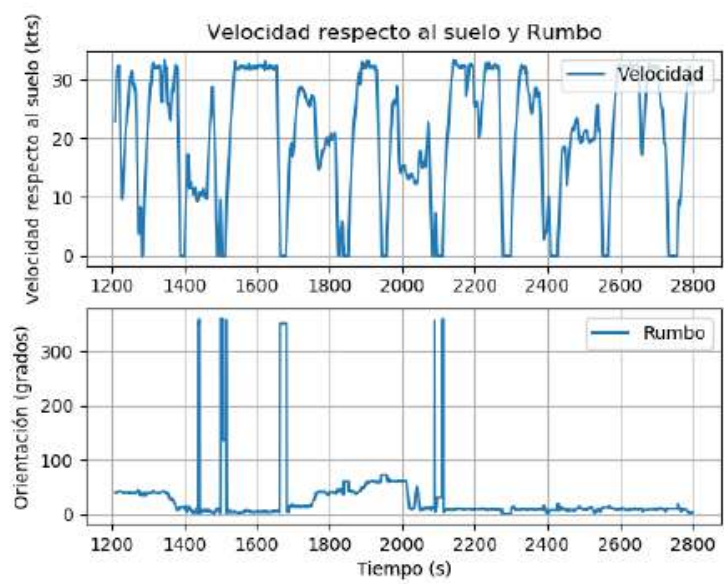

FIG. 11 Mediciones del GPS de la velocidad respecto al suelo y el rumbo del vehículo - Prueba en tierra.

por las variaciones en la presión atmosférica que se tienen entre dos puntos de medición, que son diferentes a la presión que se configuró como referencia al iniciar la prueba.

A partir de las mediciones registradas por el GPS, es posible obtener la velocidad de desplazamiento respecto al suelo y la orientación del vehículo respecto al norte magnético durante la prueba, como se muestra en la Fig. 11. Se observa que el vehículo alcanza una velocidad máxima de aproximadamente $60 \mathrm{~km} / \mathrm{h}$, y que se dirige al norte en la mayor parte del recorrido.

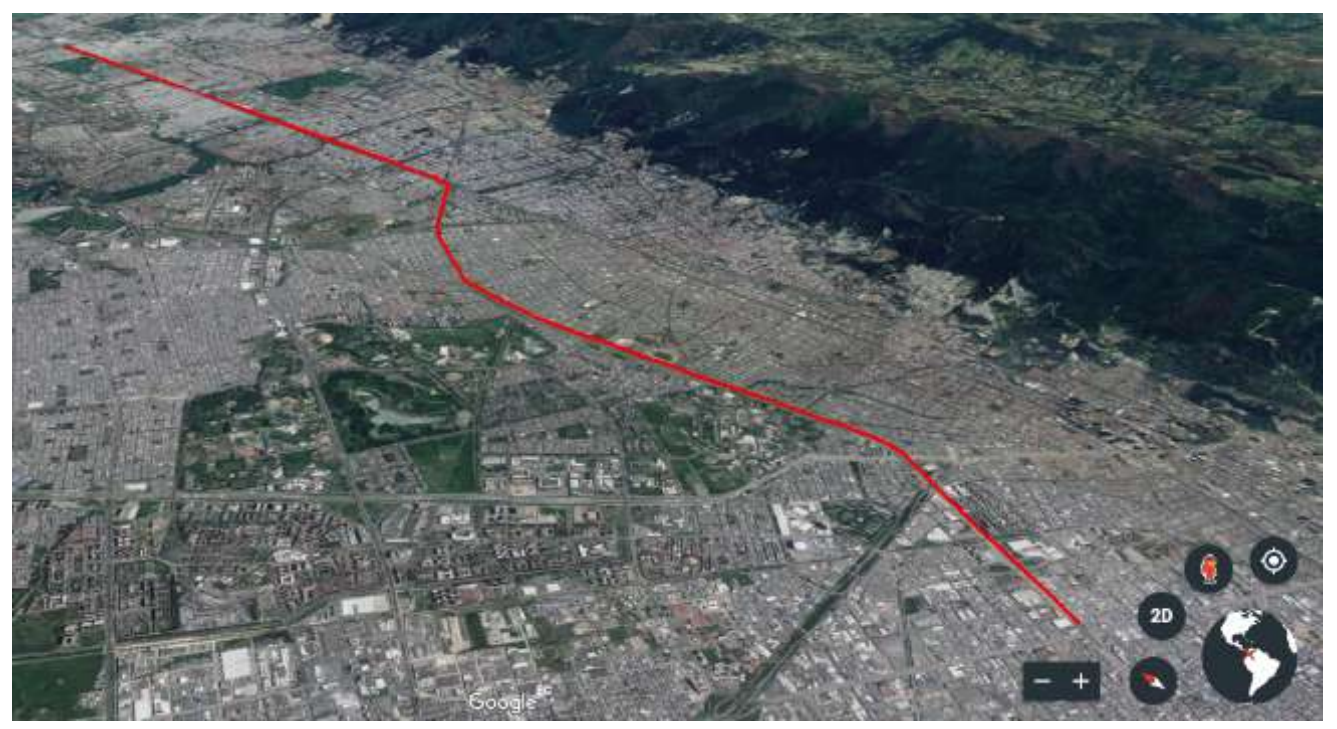

FIG. 12 Vista tridimensional del recorrido - Prueba en tierra.

Durante la fase de post-procesamiento de los datos, se generó un código para construir un archivo en formato .kml para graficar la trayectoria del vehículo en Google Earth con base en las mediciones de altitud, latitud y longitud registradas por el GPS. Así, se generó la imagen que se observa en la Fig. 12.

Del mismo modo, se realizó una prueba de medición del sistema en un vuelo comercial en la ruta Medellín - Bogotá, obteniéndose los siguientes resultados: la aceleración de la aeronave registrada por el sistema, en la dirección del movimiento, se presenta en la Fig. 13 un rango de aceleración de \pm 1 g, aproximadamente, lo cual coincide con las condiciones de aceleración que presenta el vehículo en el que se desarrolló la prueba. 


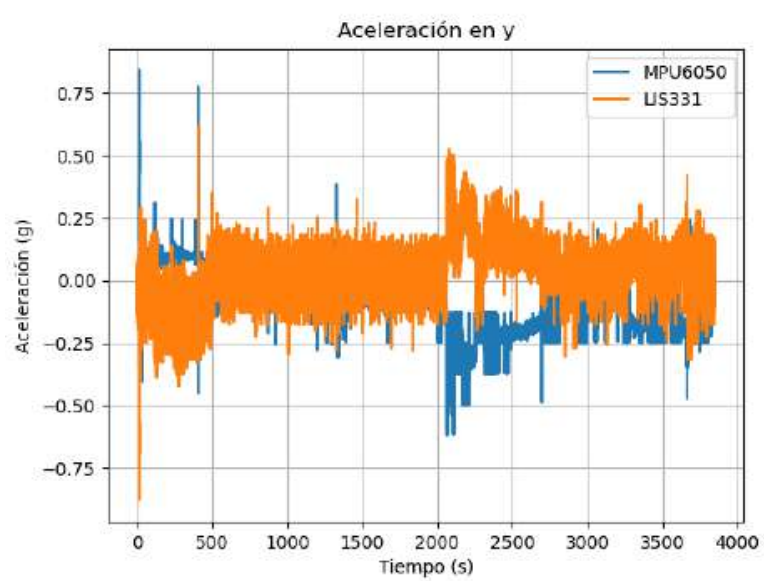

FIG. 13 Aceleración horizontal del vehículo en la FIG. 14 Mediciones de la altura de vuelo - Prueba dirección del movimiento - Prueba en vuelo comercial.

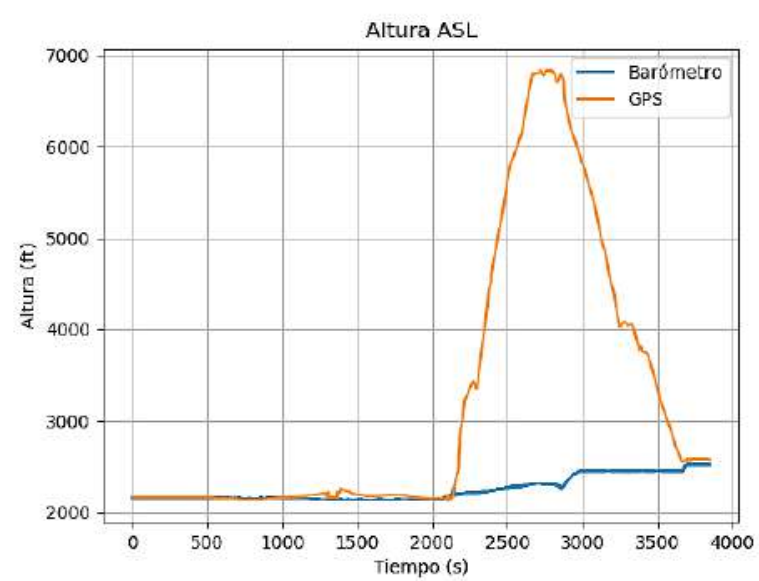
en vuelo comercial.

El barómetro y el GPS permitieron obtener las mediciones de altura sobre el nivel del mar, registradas durante el vuelo ${ }^{2}$. Se muestra en la Fig. 14 que el sistema de posicionamiento GPS registró la altura real de vuelo de la aeronave, obteniéndose una altura de crucero aproximada de 25000 pies. Por otra parte, como el sistema de adquisición de datos se llevó en la cabina de pasajeros de la aeronave, el barómetro registró la altura a partir de la presión en cabina y, por ende, no se evidencia la altura real de vuelo.

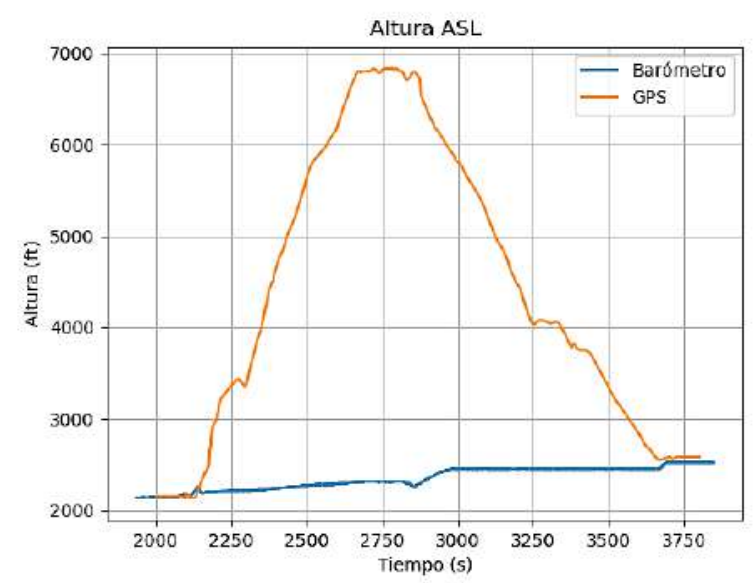

FIG. 15 Detalle de la altura de vuelo - Prueba en vuelo comercial.
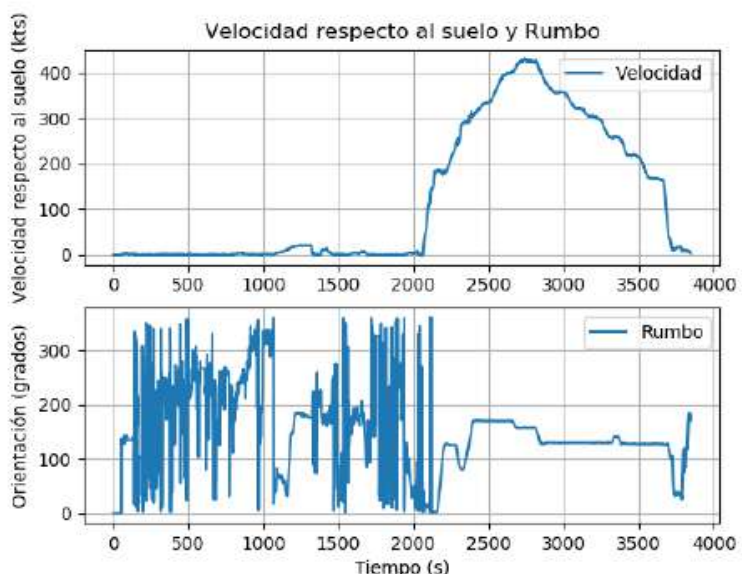

FIG. 16 Mediciones del GPS de la velocidad respecto al suelo y el rumbo del vehículo - Prueba en vuelo comercial.

En la Fig. 15 se observa un mayor detalle de las mediciones de altitud registradas. Se evidencia en primer lugar la altura de la ciudad de partida (Medellín); después, durante el ascenso, se produce una pequeña disminución en la presión en cabina, que se evidencia con el aumento en la altitud. Al finalizar la fase de crucero, se registró un ajuste en la presión en cabina, y ésta se mantiene constante hasta el aterrizaje, donde se observa una regulación con la presión de la ciudad de llegada (Bogotá).

EI GPS del sistema registró la velocidad respecto al suelo y la orientación de la aeronave, que se presentan en la Fig. 16. Se obtuvo una velocidad máxima de aproximadamente 420 nudos ( $777 \mathrm{~km} / \mathrm{h}$ ) sobre el terreno.

\footnotetext{
2 Experimentalmente, existen prototipos de radares de bajo costo que permiten mediciones de posición y velocidad como el mostrado en [20], aunque en dicho estudio las pruebas se llevaron en condiciones bastante controladas en vehículos terrestres aprovechando el efecto Doopler.
} 
Además, se evidencia la variación en la orientación durante el movimiento en la pista, y luego se observa el rumbo de la aeronave durante el vuelo.

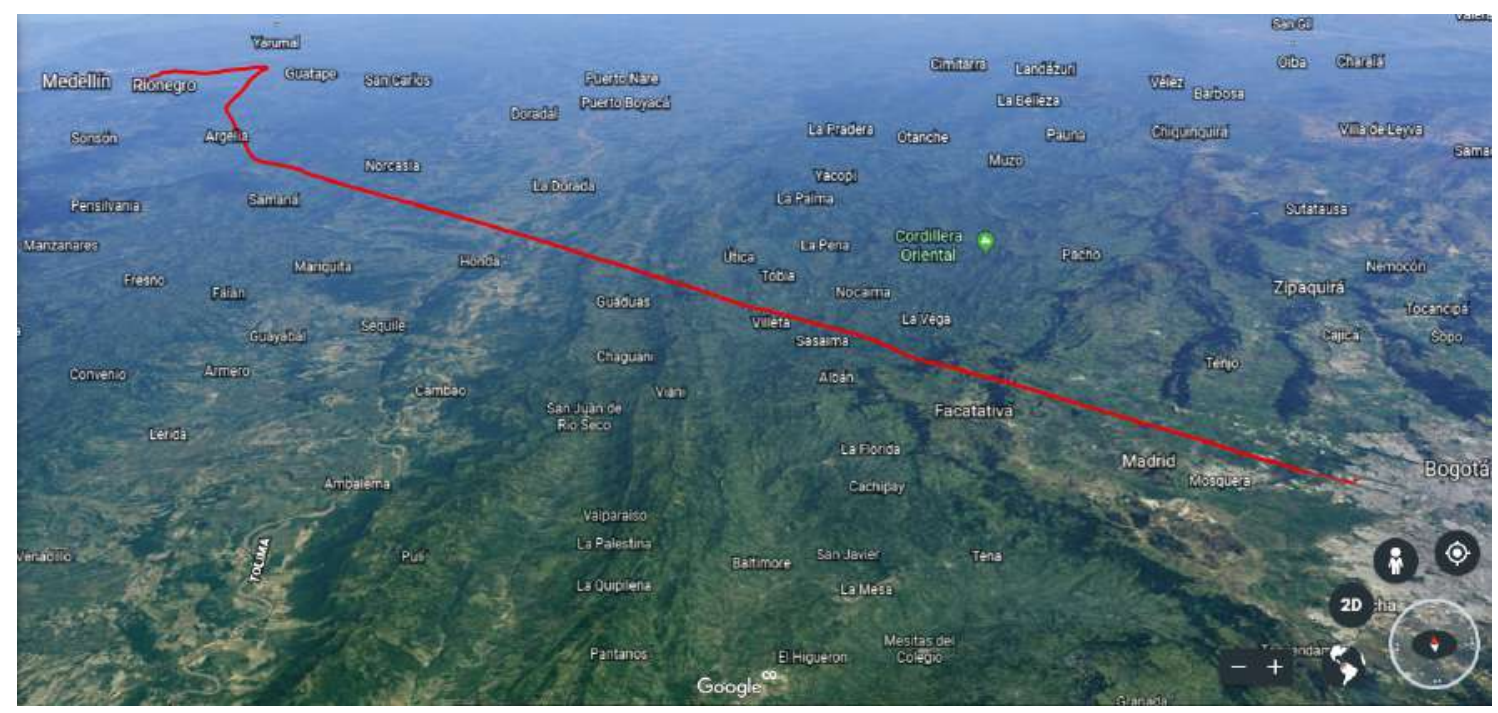

FIG. 17 Vista tridimensional del recorrido - Prueba en vuelo comercial.

Empleando la programación comentada anteriormente, se generaron las vistas del recorrido de la aeronave que se muestran en la Fig. 17.

\section{3 | CONCLUSIONES}

En este trabajo se realizó el diseño y la construcción de un dispositivo de adquisición de datos de vuelo adaptado a la geometría del cohete experimental USB - Rocket I, manufacturado por la Universidad de San Buenaventura, Bogotá D.C.

Realizando una simulación del modelo en el programa RockSim, se pudieron determinar los parámetros resultantes de vuelo del cohete empleando un motor de combustible sólido tipo J, de referencia AeroTech J270W. Se calculó en la simulación una altura de apogeo de 1965 metros, con un número de Mach máximo de 0.893, y una aceleración máxima durante el despegue de aproximadamente $18.24 \mathrm{~g}$.

Se logró realizar la integración de múltiples elementos electrónicos de medición para construir un sistema con la capacidad de obtener, almacenar y procesar variables de vuelo del cohete experimental como lo son altura, velocidad, aceleración, presión atmosférica, temperatura y posición geográfica.

Se presentaron pruebas de medición del sistema, como una prueba de desplazamiento horizontal y otra en vuelo comercial. Se verificó la sensibilidad en la medición del barómetro, y su capacidad para detectar ligeros cambios en la presión atmosférica. Además, durante las pruebas, se demostró la capacidad de funcionamiento de los acelerómetros y se comprobaron las diferentes variables medidas por el GPS.

A futuro, es necesario realizar pruebas adicionales del sistema en una plataforma capaz de simular condiciones semejantes a las de un vuelo en cohete, para finalmente realizar un vuelo del sistema en el cohete experimental USB - Rocket I. Además, se propone también la inclusión de elementos de transmisión de largo alcance, para garantizar una recepción de los datos en tiempo real durante todo el vuelo del cohete.

\section{REFERENCIAS}

[1] A. Urrego, "Investigaciones en cohetería experimental. Misión Séneca, Lanzamiento del cohete Ainkaa I," 2009. 
[2] M. G. Rincón and D. E. Rolón, "DISEÑO Y CONSTRUCCIÓN DE UNA MISIÓN DE COHETERÍA EXPERIMENTAL CON PROPELENTE TIPO SÓLIDO PARA ALCANCES ESTRATOSFÉRICOS," 2016.

[3] R. Jiménez, "Sistema de telemetría en tiempo real para cohete balístico amateur," 2009.

[4] N. Velásquez, "Diseño e implementación de centro de control e ignición de cohetes experimentales sobre un sistema embebido," Universidad de los Andes, 2013.

[5] B. Cooper, "Analysis and Design of an Affordable High Altitude Rocket System," California Polytechnic State University, Aerospace Engineering Department, San Luis Obispo, 2011.

[6] CSULA, "experimental sounding rocket: Sonic eagle"," 2013.

[7] A. Gülhan, "Main achievements of the rocket technology flight experiment rotex-t," in 21st AIAA International Space Planes and Hypersonics Technologies Conference, Hypersonics 2017, 2017. doi: 10. $2514 / 6$. $2017-2208$

[8] M. Kobald, U. Fischer, K. Tomilin, A. Petrarolo, and C. Schmierer, "Hybrid experimental rocket stuttgart: a low-cost technology demonstrator," Journal of Spacecraft and Rockets, vol. 55, no. 2, pp. 484-500, 2018. doi: $10.2514 / 1.434035$

[9] L. Zhou, Z. Sheng, Z. Fan, and Q. Liao, "Data analysis of the TK-1G sounding rocket installed with a satellite navigation system," Atmosphere, vol. 8, no. 10, p. 199, 2017. doi: 10. 3390/atmos8100199

[10] M. Christopher, T. Cojocar, and J. Deery, “Unexploded Ordnance Hybrid Rocket," 2018.

[11] Mayorga Betancourt, Manuel Alejandro and Cadavid Estrada, Juan Guillermo and Bonilla Paez, Javier Alejandro and López Santamaría, Camilo Andrés and López Gómez, Mauricio and Silva Leal, Vladimir and Galindo, José Miguel, "Use of biofuels in the aeronautical industry: Colombian air force case," Tecciencia, vol. 14, no. 26, pp. 53-63, 2019. doi: 10. 18180/tecciencia. 2019. 26.7

[12] H. Acosta and A. Urrego, "Lanzamiento, análisis y validación de datos de vuelo del cohete usb rocket-i para la obtención de la certificación tripoli de cohetería de alta potencia nivel ii. Fase II," Bogotá, 2018.

[13] C. Photo, S. Rocketry, R. Kit, and B. Milligan, "Saturn V in RockSim v9 Simulating a 2-Stage Saturn V in RockSim."

[14] H. Acosta. and A. Urrego, "Diseño de una plataforma tipo cohete para la obtención de la certificación trípoli alta potencia nivel 2," Bogotá, 2017.

[15] Bosch, "BMP180 Digital pressure sensor," 2018.

[16] Quectel, "quectel 186 compact gnss module integrated with patch antenna mt3333 solution."

[17] R. Pi, "Raspberry Pi 3 Model B+," 2015.

[18] "KiCad EDA," 2018. https: //kicad-pcb. org/.

[19] A. Augustin, "websockets: Library for building WebSocket servers and clients in Python." https: // github. $\mathrm{com} /$ aaugustin/websockets.

[20] Y. G. Parra, B. Guzmán, P. González, J. Tomás, et al., "Development of a Low-Cost, Short-Range Radar System to Measure Speed and Distance," Tecciencia, vol. 12, no. 22, pp. 99-106, 2017. doi: 10. 18180/tecciencia . 2017. 22. 11 
